\title{
So, Antidepressant Drugs have Serious Adverse Effects, but what are the Alternatives?
}

\author{
Amira Mohammed Ali ${ }^{1 *}$ and Amin Omar Hendawy ${ }^{2,3}$ \\ ${ }^{1}$ Department of Psychiatric Nursing and Mental Health, Faculty of Nursing, Alexandria University \\ ${ }^{2}$ Department of Animal and Poultry Production, Faculty of Agriculture, Damanhour University, Egypt. \\ ${ }^{3}$ Department of Biological Production, Tokyo University of Agriculture and Technology, Tokyo, Japan
}

Received Date: October 20, 2018; Published Date: November 21, 2018

*Corresponding author: Amira Mohammed Ali, Department of Psychiatric Nursing and Mental Health, Faculty of Nursing, Alexandria University, Edmon Fremon St, Smouha, Alexandria, 36741, Egypt

Abstract

Background: Pharmacological treatments of depression underwent several refinements; relatively more effective ones exist nowadays. Nonetheless, dissatisfaction with depression treatments is widespread because of the poor response and resistance that is encountered by about $33 \%$ of patients. Further, concerns are raised regarding the disabling negative adverse effects of these medications (carcinogenic, cardiotoxic, diabetogenic, etc.). A search for safer and more effective treatments is highly encouraged.

Methods: This article reviews the most relevant studies that describe adverse effects of antidepressant drugs; it also briefly explores the literature for recent reports on alternative avenues for the treatment of depression. When possible, the mechanisms underlying the addressed adverse effects and alternative treatments were detailed.

Results: All antidepressants can cause adverse effects, but SSRIs are reported to have less adverse effects in relation to TCAs and MAOIs. Furthermore, antidepressants' contribution to oxidative stress, inflammation, and cytotoxicity increase the risk of obesity, cancer, diabetes, and cardiovascular diseases in some patients. Available natural alternatives are limited and understudied to some extent. However, Omega-3 fatty acids, herbal plants, flavonoids, bee honey, probiotics, and dietary modifications seem promising approaches that can be used alone, combined with each other, or even combined with SSRIs to enhance their therapeutic effect and overcome the drug-related adverse effects.

Highlight

i. The use of antidepressant drugs has increased recently.

ii. Drug resistance prevails in around one third of the depressed patients.

iii. Neurodegeneration and cognitive dysfunction are common among depressed patients despite of receiving treatment.

iv. The adverse effects of antidepressant drugs range from minor symptoms that cause discomfort e.g., dryness of mouth to serious physical problems such as increased blood pressure and severe bleeding.

v. Antidepressant drugs contribute to colontoxicity, which reduces the production of neurotransmitters and increases microbiota related neurotoxicity.

vi. Tricyclic antidepressants and monoamine oxidase inhibitors contribute to oxidative stress and inflammation and in turn worsen the prognosis of depression and contribute to physical health problems.

Promising alternatives to antidepressants include omega-3 fatty acids, herbal plants, flavonoids, bee honey, probiotics, and dietary modifications.

Keywords: Antidepressants, Depression, Drug Resistance, Metabolic Disorders, Herbal Plants, Flavonoids, Bee Honey, Omega-3 Fatty Acids, Probiotics, Dietary Modification

Abbreviations: ECG: Electrocardiograph; ES: Effect size; TCAs: Tricyclic Antidepressants ; SSRIs: Selective Serotonin Reuptake Inhibitors; MAOIs: Mono Amino Oxidase Inhibitors; GIT: Gastrointestinal Tract; ROS: Reactive Oxygen Species; PUFAs: Polyunsaturated fatty acids; IRR: Incidence Risk Ratio; RR: Relative Risk.

\section{Introduction}

Depression is a serious and recurrent disorder, which is characterized by heterogeneous symptoms of

psychopathological sadness, inability to experience pleasure, insomnia or hypersomnia, psychomotor agitation or retardation, 
fatigue, poor concentration; feelings of hopelessness, worthlessness or guilt, and frequent suicidal ideation [1]. It results from the interaction of several pathological pathways that include genetic tendencies, intrauterine environmental factors, neurotransmitter disturbances, chronic inflammation, oxidative and nitrosative stress, gut-brain axis interactions, and environmental factors such as stress and diet $[2,3]$.

\section{An Overview of Antidepressants}

Antidepressants are considered the first line of treating depression [4]. They are also used to treat anxiety disorders (e.g., obsessive compulsive disorder) and neuropathic pain; they are used to prevent migraine[5]. Antidepressants are drugs that increase extracellular availability of monoamines [6]. They have been classified into several categories based on the patterns of neurotransmission regulation they induce. Monoamine oxidase inhibitors (MAOIs) are drugs that are less frequently used; they prevent the reuptake of biogenic amines in a nonselective fashion [7]. Tricyclic antidepressants (TCAs) are nonselective mixed serotonin and norepinephrine reuptake inhibitors that have distinct chemical structures known as tertiary amines (e.g., amitriptyline), which have greater serotonergic effect and secondary amines (e.g., nortriptyline), which have more noradrenergic effect [5]. The category of the second-generation antidepressant drugs involves drugs that selectively target one or two biogenic amines: selective serotonin reuptake inhibitors (SSRIs), serotonin and noradrenaline reuptake inhibitors (SNRIs) (e.g., venlafaxine), and the noradrenaline and dopamine reuptake inhibitors (NDRI) (e.g., bupropion).

The Newer Atypical Antidepressants (NAAs) (e.g., vilazodone) target the monoamine neurotransmitter systems similar to the other previously established antidepressants; in addition, they are thought to have a multimodal mechanism of action, which lowers the risk of sexual dysfunction that usually occur with MAOIs, TCAs, SSRIs, and SNRIs $[6,8,9]$. Based on the fact that antidepressants increase the levels of biogenic amines within hours from acute administration, and that the therapeutic effects occur after 2-4 weeks of lowers treatment, it has been hypothesized that the therapeutic action of antidepressants is mediated by intracellular signal transduction pathways. However, this hypothesis did not result in a compelling model for the action of these drug [10].

\section{Problem Statement}

The basic principle of guidelines of pharmacological treatment of depression focuses on helping patients attain remission [11]. However, it is not confirmed that the relatively trivial effect of these drugs (compared with placebo reported by clinical trials) are of clinical importance [4]. In addition, more than half the patients drop-out of treatment mainly because of adverse effects of antidepressants [12]. These drugs function mainly by targeting disturbances of neurotransmitters [6]. This solo mechanism of action leaves other depressive pathologies unaddressed. Hence, a considerable percentage of patients who receive antidepressants suffer persistence of depressive symptoms, deterioration of cognitive functions, and increased suicidality $[7,13,14]$. A current meta-analysis reported nonsignificant effect of antidepressant dose increase compared with antidepressant continuation in antidepressant resistant patients; the effect size (ES) was close to zero (ES $=0.053,95 \% \mathrm{CI}$, -0.143 to 0.248 ) [15]. Accordingly, poor overall functioning and low quality of life is wide spread among victims of depression despite of receiving treatment. [16,17].

\section{Theoretical Framework and Aim}

It seems that the literature on the efficacy and adverse effects of antidepressants is dull. A widely cited systematic review indicates selective reporting of clinical trials of antidepressant drugs that favor positive results [18]. Another recent review reports that the majority of meta-analyses that assessed the adverse effects of antidepressants were funded by manufacturers of antidepressants or conducted by employees in these companies ( $\mathrm{N}=147$ study), and these studies were less likely to report adverse effects of antidepressants compared with studies conducted by independent research entities $(\mathrm{N}=58$ study) [19]. Therefore, masking adverse consequences of these drugs would in turn negatively affect patients and the health care system as a whole.

The current review aims to uncover the most important adverse consequences of antidepressant drugs: carcinogenic effect, obesity, diabetogenic effect, and cardiotoxic effect. Whenever possible mechanisms leading to such adverse effects would be illustrated. Another purpose of this review is to explore the literature for safer evidence-based alternatives-this review focused on interventions for which we could find review studies that reported positive effects such as omega-3, herbal plants, diet, and other alternative treatments for depression that involve oral intake, which is easier to take part in than long-term active treatments such as CBT, yoga, exercise, or other sophisticated procedures such as deep and synchronized transcranial magnetic stimulation. To achieve the aim of this paper a literature search of PubMed and Google Scholar was conducted using terms directly related with the targeted outcomes (adverse effects of antidepressants and alternative antidepressant treatments) to obtain the relevant research articles. A variety of studies were consulted: RCTs, systematic reviews, meta-analyses, observational studies, in addition to in vitro and animal studies.

\section{Limited Efficacy of Antidepressants}

Antidepressants have been prescribed to treat depression for several decades; their prescription rate has even increased in the last few years [19]. Yet, the burden of depressive disorders is increasing, which sets the efficacy of these drugs questionable [20]. A former meta-analysis reported no significant differences for remission and premature treatment discontinuation between antidepressants and placebo in long-term (treatment duration 6-8 months) parallel-arm efficacy trials [21]. A recent cohort study that involved repeated assessment of symptoms in 
a representative community sample of 4,547 patients over 30 years indicated that antidepressant use is associated with poorer long-term outcomes of depression relative to non-use [20].

Recent evidences indicate that antidepressant drugs may contribute to the deterioration of the prognosis of depression. The mechanism of action of these drugs negatively interferes with other several pathological pathways of depression. The effect starts locally from their intake sites in the gastrointestinal tract (GIT), which can affect both the neurotransmitters pathway and the gut-brain axis. In addition, their systemic effects involve activation of oxidative stress and inflammation, which enhance neurotoxicity (Figure 1).

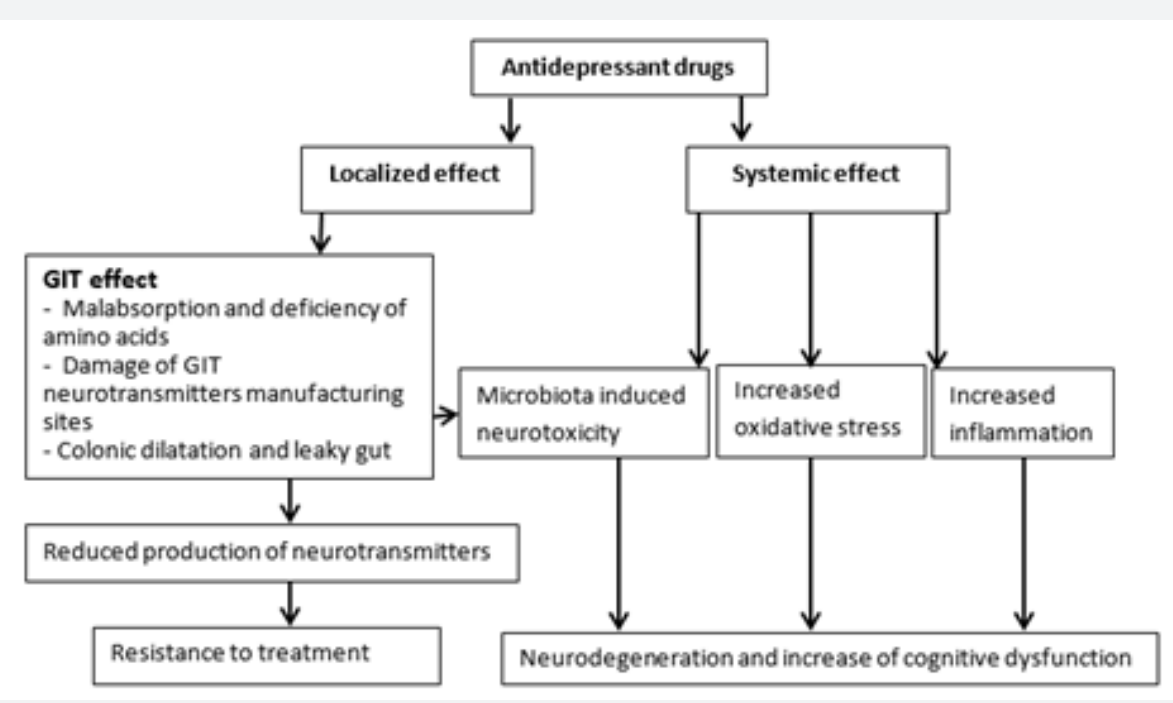

Figure 1: Effect of antidepressant drugs on the prognosis of depression.

\section{Localized Effect on The GIT: Activation of The Gut- Brain Pathway}

Some antidepressants such as TCAs have been reported to exert cytotoxic effects on the GIT by antagonizing prokinetic neurotransmitters, stimulating antikinetic neurotransmitters, relaxing smooth muscle, promoting dysmotility, and injuring enteric neurons. Colonic pseudo-obstruction is a form of antidepressants induced colonotoxicity, which is characterized by diffuse dilatation of the intestinal loops containing multiple air-fluid levels. It manifests with symptoms of constipation, abdominal pain, distention, etc. If untreated it progresses into necrotizing enterocolitis and colonic ischemia [22] . Colonotoxicity leads to further exacerbation of depression in a number of ways. On one hand, mal-absorption takes place as a consequence of drugs-induced GIT toxicity, which results in deficiency of the nutritional ingredients (amino acid precursors e.g., tryptophan and tyrosin) necessary for the formation of all neurotransmitters. Further, it is reported that $95 \%$ of serotonin is produced in the endocrine cells of the GIT; inflammation induced destruction of the GIT signals serious deficiencies in the body's serotonin formation infrastructures. It goes without saying that serotonin is the main neurotransmitter responsible for the regulation of mood [23]. In addition, drugs-induced GIT inflammation increases the possibility of leaky gut. A recent systematic review indicates that antidepressants have an antimicrobial activity, which alters microbiota composition and induces gut permeability [24]. Hence, the normally existing microflora get to the circulation, release their toxins (e.g., lipopolysaccharides produced by gram-negative bacteria). These processes, in turn, increment oxidative stress and inflammation, and causes serious neurotoxic effects [25].

\section{Systemic Effect: Oxidative Stress, Inflammation, and Immune Modulation}

The therapeutic effect of antidepressant drugs involves increasing levels of biogenic amines. On the contrary, the interaction between the monoamine oxidases (MAOs) and their monoamine substrates results in several byproducts that activate oxidative stress and further increases the turnover of biogenic amines. This strategy contributes to the generation of neurotoxic aldehydes, hydrogen peroxide, reactive oxygen species (ROS), increased 5-hydroxyindoleacetic acid/5-HT ratio, and nuclear factor- $\kappa \mathrm{B}(\mathrm{NF}-\kappa \mathrm{B})$ activation. The effect of such oxidative stress and its associated inflammatory processes leads to alterations of neuroplasticity and neurotrophins in selective vulnerable cerebral areas, which is linked to alterations in mitochondrial function and neuronal bioenergetics, DNA damage, aptosis, and cell death. Such processes accelerate the course of neurodegeneration among depressed patients $[10,14]$. A study that tested the effect of 4 antidepressants amitriptyline, clomipramine (TCAs), citalopram and paroxetine (SSRIs) on the immune response of abalone hemocytes in vitro, reported that the four antidepressants exhibited different cytotoxicities. A significant dose-dependent stimulation of the hemocyte immune efficiency was noticed: destabilization of lysosomal membranes, phagocytosis, and production of reactive oxygen 
species increased at lower concentrations but decreased at higher concentrations. TCAs affected the immune response more strongly than SSRIs [26].

\section{Adverse Effects of Antidepressants}

Despite the marginal benefits they produce compared with placebo, antidepressants contribute to a wide range of psychological and biological adverse effects. In addition to the stigma associated with the prolonged use of these drugs, interpersonal adverse effects of antidepressants that involve aspects of sexual life, social life, work or study are high, up to $52 \%$ of participants of a former study [27]. This is probably associated with the high treatment drop out that in most instances takes place without consulting the physician [28]. A recent metaanalysis reports that different antidepressants (even within the same generation) vary considerably according to their efficacy, acceptability, and tolerability [29]. The biological adverse effects of antidepressants are also heterogeneous; they range from simple and tolerable side effects such as photosensitivity and mouth dryness to serious and life threatening complications such as cataract, arrhythmia, and intracranial hemorrhage [30-32]. Therefore, it is clear that antidepressant drugs may contribute to the pathologic mechanisms of several serious disorders. This manuscript sheds light on 4 of these conditions: cancer, obesity, diabetes, and cardiovascular diseases. Figure 2 illustrates the mechanisms through which antidepressants maybe associated with these disorders. The pathological mechanisms through which antidepressants produce adverse effects are related mainly to oxidative stress, immunomodulation, inflammation, and homeostatic imbalance [33-35]. However, several factors interact with the action of these drugs (the degree of depressive symptoms, presence of comorbidities, age, general condition, stress, diet, etc.), which may enhance or counteract their negative effects $[32,36]$.

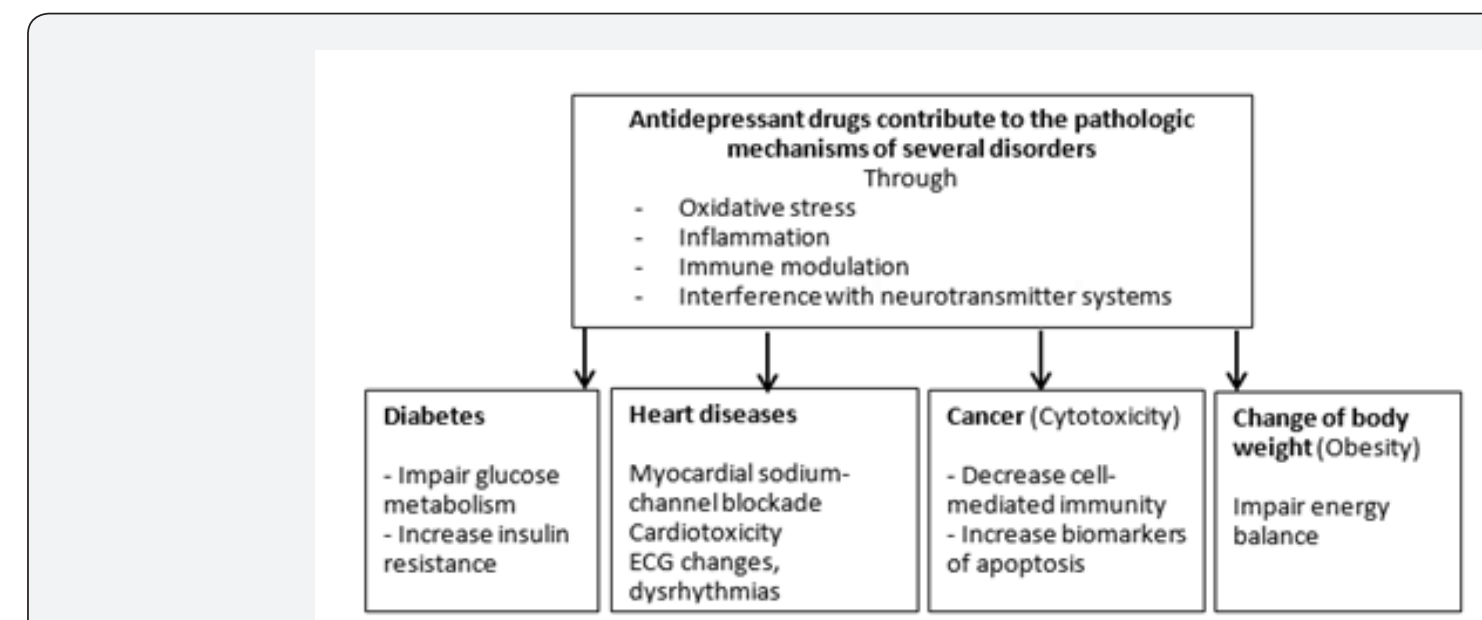

Figure 2: Antidepressants-related disorders and their associated mechanisms.

\section{Antidepressants and Risk Of Cancer}

Antidepressants are used to treat depressive symptoms in cancer patients; however, a recent Cochrane review reports no significant antidepressant effect of these drugs compared with placebo [37]. Furthermore, antidepressants are frequently prescribed concurrently with opioids in cancer patients to enhance their effect of neuropathic pain reduction [38]. However, such combinations with anti-cancer drugs (e.g. platinum) have synergistic cytotoxicity effect evidenced by increased biomarkers of apoptosis compared with cytotoxicity induced by solo anti-cancer [39]. A former systematic review and a recent meta-analysis reported that combining antidepressants with opioids in cancer is less likely to reduce pain intensity of greater than 1 point on a $0-10$ numerical rating scale; the effect size for all drugs was much smaller than that in non-cancer patients with neuropathic pain. Meanwhile, such combination is associated with an increase in adverse events $[38,40]$.

Several animal studies indicate that antidepressant drugs (TCAs, MAOIs, and SSRIs) stimulate carcinogenesis and promote the growth of neoplasms [41-43], even when administered at therapeutic doses [44]. Similarly, a number of large scale observational studies support an association between intake of antidepressants and incidence of different types of cancer [4548]. However, TCAs and MAOIs are associated with higher risk than SSRIs [45]. The overdose of certain drugs e.g., bupropion encompasses a higher risk [49]. The mechanism involves dampening natural killer cell function and cell-mediated immunity ( $\mathrm{T}$ and $\mathrm{B}$ lymphocyte proliferative responses) $[35,41]$. It is suggested that these drugs enhance tumor growth by binding to growth-regulatory intracellular histamine receptorsassociated with anti-estrogen binding sites in microsomes and nuclei-stimulating catalytic activity of cytochrome P450 monooxygenases and inhibiting their binding to histamine receptors $[43,44]$.

\section{Antidepressants and Risk of Obesity}

Use of antidepressants has been reported to cause considerable weight gain [50]. Nonetheless, antidepressants vary considerably in their association with weight gain [2]. Some antidepressants induce a weight gain of $20 \mathrm{~kg}$ over several months of treatment [51]. Obesity increases the risk of several 
related disorders such as diabetes mellitus, cardiovascular diseases, cancer, etc., which lowers life expectancy and increases mortality [52]. Nonetheless, the association of antidepressants with changes of body weight varies considerably based on the type and dose of the used drug as well as individuals' characteristics [2,51,53]. A large meta-analysis study reported that amitriptyline, mirtazapine, and paroxetine were associated with a greater risk of weight gain. On the other hand, SSRIs e.g., fluoxetine caused weight loss during the acute phase of treatment [51]. Sibutramine is an antidepressant that is widely used as an appetite suppressant, not as antidepressant in depression treatment. It effectively promotes weight loss (about 4-5 kg); however, its use is associated with an increase of blood pressure and pulse rate $[54,55]$. Phentermine, an appetite suppressant, is sometimes used in combination with antidepressants to counteract their negative effects on body weight; however, concerns are raised about its co-administration [56]. A large controlled trial that recruited 2487 obese participants who received a combination of phentermine and topiramate to lower their body weight indicated some acceptable levels of weight loss. Nonetheless, a trail of adverse effects was reported: dry mouth, constipation, insomnia, paraesthesia, depression-related and anxiety-related adverse events [52], which indicates that antiobesity drugs interfere with the regulation of mood.

Several complex mechanisms are involved in the regulation of energy balance and body weight. Drugs that cause body weight change interfere with a number of neurotransmitter systems (e.g., antagonism of the 5-HT2C receptors) that act in several hypothalamic nuclei to regulate the stores of body fat $[36,53]$. One possible mechanism involves inhibition of the gene expression of several enzymes that are essential for the transcriptional regulation of adiponectin-a protein derived from adipocytes, which regulates insulin sensitivity and glucose homeostasis. This consequently leads to obesity that is associated with insulin resistance [33]. The anorexiant role of these drugs involves reduction of appetite, induction of satiety, and thermogenesis [54]. Given the fact that all trials of antiobesity drugs (including antidepressants e.g., sibutramine) have been limited by their high attrition rates and lack of longterm morbidity and mortality data, the safety of these drugs is not established especially as thermogenesis is associated with enhancement of oxidative stress [55].

\section{Antidepressants and Risk of Diabetes}

Use of antidepressants has been associated with both hyperglycemic and hypoglycemic effects [2]. Serotonin plays a vital role in glucose regulation; most antidepressants increase the neurotransmission of serotonin. In particular, antagonism of 5-HT2C receptors and M3 muscarinic receptor is associated with obesity (a risk factor of diabetes) and diabetogenic effects: reduced insulin secretion from beta cells due to inhibition of cyclic adenosine monophosphate (cAMP) in the pancreas, reduced sensitivity to insulin, and impaired glucose tolerance $[33,36]$.
In an in vitro study that tested the effect of 3 antidepressants on murine and human cell-line models of pancreatic $\beta$-cells, antidepressants (even at therapeutic doses) decreased the redox, oxidative respiration, and energetic potential of $\beta$-cells. This effect was associated with inhibition of mitochondrial complex I and III, increased lactate output, and decreased insulin secretion. Chronic administration of antidepressants increased oxidative stress and activated caspases, 3, 8, and 9 [34].

A Diabetes Prevention Program that followed 3187 participants on 3 treatments (intensive lifestyle, metformin, and placebo) for an average of 3.2 years reported a significant diabetogenic effect of continuous and intermittent use of antidepressants in the placebo and the intensive lifestyle groups compared with no use of antidepressants; hazard risks ranged between 2.25 and 3.48 [57]. A former review reported that noradrenergic antidepressants and TCAs impair the metabolism of glucose in non-diabetics and cause deterioration of the metabolic situation in diabetics. Meanwhile, SSRIs are thought to improve glucose regulation in the short run although their long term effect is under dispute [58]. However, the effect may depend on the dose and duration of administration. A large scale nested case-control study identified the incidence of 2,243 cases of diabetes mellitus in a cohort of 165,958 depressed patients who received at least one new prescription of an antidepressant. Compared with no use of antidepressants, use of TCAs and SSRIs for more than 2 years in moderate to high daily doses was associated with an increased risk of diabetes (IRR $=1.77,95 \%$ $\mathrm{CI}=1.21-2.59)$ and ( $\mathrm{IRR}=2.06,95 \% \mathrm{CI}=1.20-3.52)$, respectively. Meanwhile, no risk was associated with treatment with lower daily doses or for shorter periods [50].

\section{Antidepressants and Risk of Cardiovascular Diseases}

Several review reports indicate that TCAs and MAOIs cause cardiotoxicity i.e., they induce a variety of cardiovascular disorders and worsen the prognosis in established cardiovascular conditions [59-61]. Combined use of antidepressants and heart medications, especially in recent cardiac conditions, increases the risk of recurrence and cardiac re-hospitalization $[8,61]$. TCAs e.g., imipramine at therapeutic doses are associated with electrocardiographic (ECG) changes that involve T-wave changes, abnormally prolonged PR and QRS intervals [62]. Such ECG changes can be diagnostic of TCAs toxicity [63]. Further, drugs such as reboxetine, duloxetine and venlafaxine can increase blood pressure [64]. A big cohort study reported an association between use of TCAs with an increased risk of myocardial infarction (RR=2.2, 95\% CI 1.2 to 3.8) compared with no use of antidepressants in an analysis that was adjusted for age, gender, baseline heart disease, diabetes, hypertension, hyperlipidemia, anxiety, and cancer [60].

The cardiotoxicity induced by antidepressants results mainly from myocardial sodium-channel blockade and autonomic function changes, which result in cardiovascular collapse: 
ventricular dysrhythmias, decreased cardiac output and cardiac contractility, changes in platelet activity, hypotension or hypertension-peripheral vascular resistance increases as a compensatory mechanism to maintain blood pressure $[30,62,64]$. TCAs, especially at high doses, are associated with sudden death - a characteristic feature of cardiovascular disease [62]. In line, a 3-year period medicolegal autopsy survey in Finland indicated that sudden death of 49 persons was associated with the use of antipsychotic and antidepressant drugs [65]. It is noteworthy that the cardiac effects of individual antidepressant drugs vary considerably; some antidepressants (e.g., fluoxetine, citalopram, bupropion) have neutral effects and are considered safe to use in patients with established coronary artery disease [64]. SSRIs are thought to have cardio-protective effects as they inhibit the reuptake of serotonin into thrombocytes which affects platelet reactivity and endothelial reactivity $[31,66]$. However, a number of meta-analyses indicate that such property of SSRIs increases the risk of severe bleeding and stroke $[31,46,66,67]$.

\section{Evidence-Base Effective Alternatives to Antide- pressants}

It is equally important that the potential benefits of antidepressant treatments outweigh their potential adverse effects. Thus, there is an increased need for safe and creative treatments that can target different neurobiological systems in order to restore functioning, promote resilience, and reduce the long-term vulnerability to recurrence of depressive episodes [7]. Evidence notes that metabolic and dietary factors play a major role in the pathology of depression [68]. Therefore, the new trends in depression management have recently moved from the use of synthetic drugs toward the use of natural and safer alternatives that can be easily incorporated into daily regimenmaking food our medication. A recent review reported that a large number of nutrients can exert antidepressant effects such as long-chain omega-3 fatty acids, magnesium, potassium, selenium, thiamine, vitamin A, vitamin B6, vitamin B12, vitamin C, and zinc [69]. In this respect, we searched PubMed and Google Scholar for studies on omega-3, herbal plants, diet, and the like. We selected treatments for which we could find review studies in the available literature that reported positive effects. Herein, we discuss some of these treatments.

\section{Omega-3 Polyunsaturated Fatty Acids}

Long-chain omega-3 polyunsaturated fatty acids (PUFAs) exist in fish oils. PUFAs are of importance to people with mood disorders since their metabolism in the brain results in several active products. Of particular interest the eicosanoids, which is a group of oxygenated C20 compounds that include prostaglandins, thromboxanes, leukotrienes and a variety of hydroxy and hydroperoxy fatty acids [70-72]. These products act in the intracellular environment as neuronal secondary messengers; they enhance cellular activity and preserve membrane fluidity by decreasing the level of cholesterol. They are involved in cholinergic, serotoninergic and catecholaminergic synaptic transmission. They contribute to neuromodulation, synaptic plasticity, and synaptogenesis mainly by interacting with extracellular G-protein-coupled receptors on neurons and glial cells. PUFAs positively influence cell migration and apoptosis [70]. Several meta-analyses have reported significant antidepressant effect of omega-3 fatty acid treatment [71-74].

Evidence indicates that omega-3 fatty acids work best in cases with a clinical diagnosis of depression not in healthy people who may experience depressive symptoms [73,74]. Interestingly, concurrent use of PUFAs with antidepressants (fluoxetine or mirtazapine) at both therapeutic and low ineffective doses induced additive antidepressant effects in rats [75]. Combining PUFAs with citalopram has been reported to speed the initial antidepressant response compared to solo antidepressant treatments [71]. Further, use of low dose of eicosapentaenoic acid, a common PUFA, as an adjunctive treatment in patients with mild-to-moderate depression who were non-responsive to medication resulted in better response to antidepressants as evidence by significant lowering of depression scores [76]. In addition, PUFAs can be beneficial for obesity and the metabolic side effects of antidepressants [71]. However, PUFAs may exhibit few negligible risks [71,72].

\section{Herbal plants}

Herbal plants are polyphenols that possess strong antioxidant properties [77]. They positively affect depression, and they have minimal adverse effects compared with conventional antidepressants [1]. Animal studies indicate that curcumin increases brain derived neurotrophic factor levels and activates the signaling pathways of 5-HT1A/cAMP/PKA/CREB [78,79]. In two clinical trials involving depressed human subjects curcumin was effective reducing depressive symptoms comparable to antidepressants $[77,80]$. Similarly, a hydro-alcoholic extract of Nigella sativa was successfully used to treat and prevent LPSinduced depression-like behaviors in mice [81]. Further, Nigella sativa was reported to provide a DNA protection under use of cytotoxic agents [82].

Terpene constituents of ginger's extract have moderate inhibitory properties against the MAO-A enzyme in vitro [83]. Cinnamon has been noted to affect regulatory proteins antioxidant and antiproliferative activity in a positive fashion. Cinnamon extract also has antihyperglycemic and antihyperlipidemic effects [84]. A recent systematic review indicates that black cohosh, chamomile, chasteberry, lavender, passionflower, and saffron have the most favorable risk-benefit profiles for anxiety and depression; these herbs may benefit cancer patients in particular by minimizing medication load and side effects [1]. Despite claims that herbal plants might be contaminated with toxic substances e.g., Pyrrolizidine alkaloids, a recent risk-benefit analysis review indicates that quantified Saint John's Wart extract is a safe and effective treatment option, and its potential of treating depression outweighs the hypothetical risk of its contamination [85]. It is worth noting 
that more rigorous RCTs are needed to establish the efficacy of antidepressant herbs.

\section{Flavonoids and glycosides}

Flavonoids and glycosides are natural products that can be used to treat depression. They are naturally occurring metabolites that exist in a variety of herbal plants, fruits, vegetables, bee products (e.g., propolis and honey), and other natural products. They confer color, flavor, and aroma, as well as nutritional and health benefits $[14,17,86]$. A large cohort study followed 82,643 midlife women (with no history of depression) for 10 years reported that intake of total flavonoids and subclasses-flavonols, flavones, flavanones, anthocyanins, flavan-3-ols, polymeric flavonoids, and proanthocyanidinswas significantly associated with lower risk of depression (HR = 0.83; 95\% CI: 0.77, 0.90) [87].

Polyphenol flavonoids exhibit several pharmacological properties since they affect various physiological and biochemical functions in the body. A former review supports the antidepressant activity of several flavonoids such as Chrysin, rutin, quercetin, and hesperidin. Their therapeutic effect ensues from a variety of biological activities such as altering oxidative/ antioxidant defenses and altering inflammatory responses [14]. Evidence supports their action of inhibiting monoamine oxidases; however, individual flavonoids vary according to the monoamine oxidase they target. Of particular note are results from two animal experiments suggesting that flavonoids isolated from the whole plant of Viola odorata L decreased depressive-like behaviors by interacting with the serotonergic system (5HT1A, 5HT2A, and 5 HT3 receptors), and in particular the 5 HT3 receptor subtype [88]. Meanwhile, the other model reported an antidepressant effect of flavonoids in Scutellariae Radix, and the mechanism involved activation of the nigra-striatal dopaminergic system and brain reward center [89]. A current review of preclinical studies indicates that glycosides exert their antidepressant effect by modulating the brain-derived neurotrophic factor (BDFN) in the hippocampus, which promotes synaptic efficacy, neuronal connectivity and neuroplasticity [88]. Further investigations of the antidepressant effect and mechanism of flavonoids and glycosides is warranted.

\section{Bee Honey}

Bee honey has been proposed as an effective treatment of depression. Honey is rich in several components known to counteract oxidative stress and inflammation e.g., flavonoids, steroid, alkaloids, saponins, tannins, etc. [90,91]. It has an inhibitory effect on more than 60 species of bacteria: aerobes and anaerobes, Gram-positives, and Gram-negatives [92,93]. Thus, it can also act through the gut-brain axis by treating gut leakage given that it possesses proven gastroprotective effects $[94,95]$. In addition, honey can exert its effect directly on the gut microbiota, which extends its benefit through the gut-brain axis' neuronal, humoral and the cell signaling. Of interest, mice treated with aflatoxin that received bee honey experienced a significant increase of the colon counts of bifido bacteria and lactobacilli-microbiota that are known as protective against depression [96]. Similarly, honey can limit the growth of harmful microflora which support a balanced microbiota [92]. A recent review that summarized findings from animal and human studies that involved use of different types of bee honey to treat depression revealed that administration of honey lowered depressive symptoms in humans and depressive-like behaviors in animals [17]. Animal studies portrayed positive effects of honey on biomarkers of depression in the blood, brains and livers of the treated animals: the levels of serum ACTH, corticosterone levels, oxidation and antioxidation markers, brain-derived neurotrophic factor [97], and monoamines in the liver [98]. Given the fact that human trials that tested the effect of bee honey are limited, it is necessary to examine the antidepressant effect of honey and its phenolic components in larger and more representative samples.

\section{Probiotics}

Probiotics are treatments that target the gut-brain pathway either by implanting live microorganisms in specific doses or nurturing beneficial gut microflora to produce health benefits to the host-Lactobacilli and Bifidobacteria are the most commonly used $[99,100]$. Intestinal microbiota produce their beneficial effects by increasing mucin expression which increases stability of the mucosal barrier, inhibiting over growth of pathogens, stimulating mucosal immunity (secretory $\operatorname{IgA}$ ), activating xenobiotic metabolism system, and synthesizing beneficial substances such as antioxidants, vitamin $\mathrm{K}$, and shortchain fatty acids [99].

In an experimental model, treatment of stressed mice with a combination of fructo-oligosaccharides and galactooligosaccharides for 3 weeks reduced stress-induced corticosterone and proinflammatory cytokine levels as well as depression-like and anxiety-like behavior. Probiotics normalized the effects of stress on the microbiota; it positively affected short-chain fatty acid concentrations e.g., increased cecal acetate and propionate and decreased isobutyrate concentrations. It also modified specific gene expression in the hippocampus and hypothalamus [101].

Probiotics use in humans is limited, but results are promising. A recent meta-analysis that evaluated the effect of probiotics on depressive symptoms revealed no effect of probiotics on mood. A subgroup analysis indicated significant improvements in the mood of individuals with mild to moderate depressive symptoms compared with healthy adults. It is worth noting that subjects in most of the included studies were healthy and the meta-analysis addressed no outcomes other than mood change [100]. A double blind RCT examined the effects of supplementing probiotic (Lactobacillus acidophilus, Lactobacillus casei, and Bifidobacterium bifidum) to depressed patients for 8 weeks. Probiotic treatment significantly decreased depressive 
symptoms, serum insulin levels, homeostasis model assessment of insulin resistance, and serum high sensitivity C-reactive protein concentrations compared with the placebo. Probiotics positively affected level of antioxidants, which was evidenced by a significant elevation of plasma 53 total glutathione levels [102]. Further investigations of the antidepressant effects of probiotics would be of value.

\section{Nutritional Modification}

A recent systematic review indicates that certain foods can effectively prevent and treat depression such as sea foods, meat of animal organs such as liver, green leafy vegetables, lettuces, peppers, and cruciferous vegetables [69]. While consuming healthy diet (e.g., Mediterranean diet) is associated with decreased odds for depression, unhealthy (sugar and fat-rich) diet can dramatically alter the composition of the gut microbiota and heighten the risk of depression [103,104]. Consuming a healthy diverse diet promotes more diversity of gut microbiota [105]. Thus, use of diet to modify the gut microbiome can be helpful in preventing and treating depression [103]. In this respect, a significant association has been found between habitual consumption of the Mediterranean diet and Prevotella genus-indicative of the microbiome family of Prevotellaceae, which is considered protective against depression [106]. In fact, food can be not only a source of amino acids that are needed for neurotransmitter formation but also a source of probiotics: around $35 \%$ of all lactic acid bacteria that exist in raw fruits and vegetables can survive gastric conditions [99].

\section{Discussion}

This review focused on investigating the carcinogenic effects, obesity, diabetogenic effects, and cardiotoxic effects of antidepressants. Despite availability of pharmacological treatment guidelines, the literature highlights inadequate recognition and treatment of the adverse effects of antidepressant drugs $[11,12]$. It is reported that more than half of those who use antidepressants discontinue treatment because of lack of efficacy and adverse effects such as weight gain and sexual dysfunction $[107,108]$. Treatment discontinuation in more than half the patients takes place without consulting the prescribing doctor [28], which indicates a communication gap and ignorance of health professionals of patients' perceptions and painful experiences related to prolonged use of antidepressants. In addition, patients who receive these medications are rarely screened for cardiac problems, diabetes or dyslipidemia; clinicians' lack of knowledge regarding the adverse effects of these drugs is considered a main factor [33].

Both lack of efficacy of antidepressants and their vast adverse consequences necessitate the search for other effective treatments that can be more acceptable. In our search, we selected natural interventions for which we could find review studies that reported positive effects. We retrieved a bunch of meta-analyses and systematic reviews on the antidepressant effects of omega-3 fatty acids [71-73,76], herbal plants (e.g.,
Saint John Wart) $[1,85]$ and their extracts such as flavonoids and glycosides [86]. Strong evidence supports the antidepressant effect of these nutrients. We formerly reviewed the literature for the effect of bee honey on depression both in humans and in preclinical models, and therefore included honey on the list [17]. What is reassuring about most of the offered treatments is that they are natural products that are in most instances foods that are safe. Nonetheless, some of these treatments such as probiotics are under-tested-though investigated in animal models, few human studies are available. Therefore, further efficacy researches concerning the offered treatments are necessary.

\section{Strengths and Limitations}

One of the merits of this study is that it involved a wide range of evidence that ranged from metanalyses of well-designed randomized trials to animals and in vitro studies; the latter allow manipulations and investigation of vital outcomes that cannot be explored in the most rigorous randomized trials. Hence, this review might represent a full description of the current state of knowledge of adverse effects of antidepressants and the available alternative treatments. Nonetheless, for lack of fund we could not do a systematic review since systematic search, screening, and extraction of data are rather costly. So, we alternatively discussed the topic in a narrative fashion.

\section{Conclusion}

Most antidepressants drugs-from all categories and generations-exhibit a range of adverse effects that differ from one medication to another. Furthermore, antidepressants' contribution to oxidative stress, inflammation, and cytotoxicity leads to deterioration of the prognosis of depression and contributes to an array of highly morbid health problems in some patients. Available natural alternatives are limited and understudied to some extent. However, omega-3 fatty acids, herbal plants, flavonoids, bee honey, probiotics, and dietary modifications seem promising approaches that can be used alone, combined with each other, or even combined with SSRIs to enhance their therapeutic effect and overcome the drug-related adverse effects. Further rigorous investigations are needed to test the cost-effectiveness of these alternatives.

\section{References}

1. Yeung KS, Hernandez M, Mao JJ, Haviland I, Gubili J (2018) Herbal medicine for depression and anxiety: A systematic review with assessment of potential psycho-oncologic relevance. Phytother Res 32(5): 865-891.

2. Holt RI, de Groot M, Golden SH (2014) Diabetes and depression. Curr Diab Rep 14(6): 491.

3. Wigner P, Czarny P, Galecki P, Sliwinski T (2017). Oxidative and Nitrosative Stress as Well as the Tryptophan Catabolites Pathway in Depressive Disorders. Psychiatr Danub 29(4): 394-400.

4. Linde K, Kriston L, Rucker G, Jamil S, Schumann I, Meissner k, et al. (2015) Efficacy and acceptability of pharmacological treatments for depressive disorders in primary care: systematic review and network meta-analysis. Ann Fam Med 13(1): 69-79. 


\section{Novel Approaches in Drug Designing \& Development}

5. Hicks J, Sangkuhl K, Swen J, Ellingrod V, Müller D, et al. (2017) Clinical pharmacogenetics implementation consortium guideline (CPIC) for CYP2D6 and CYP2C19 genotypes and dosing of tricyclic antidepressants: 2016 update. Clinical Pharmacology \& Therapeutics 102(1): 37-44.

6. Leuchter AF, Hunter AM, Krantz DE, Cook IA (2015) Rhythms and blues: modulation of oscillatory synchrony and the mechanism of action of antidepressant treatments. Annals of the New York Academy of Sciences 1344(1): 78-91

7. Caraci F, Calabrese F, Molteni R, Bartova L, Dold M, et al. (2018) International Union of Basic and Clinical Pharmacology CIV: The Neurobiology of Treatment-resistant Depression: From Antidepressant Classifications to Novel Pharmacological Targets. Pharmacol Rev 70(3): 475-504

8. Biffi A (2017) Antidepressants and the risk of cardiovascular diseases. (Doctorate), Università Degli Studi Di Milano-Bicocca.

9. Hillhouse TM, Porter JH (2015) A brief history of the development of antidepressant drugs: From monoamines to glutamate. Experimental and clinical psychopharmacology 23(1): 1-21.

10. Ferrari F, Villa RF (2017) The Neurobiology of Depression: an Integrated Overview from Biological Theories to Clinical Evidence. Molecular Neurobiology 54(7): 4847-4865.

11. Davidson JR (2010) Major depressive disorder treatment guidelines in America and Europe. J Clin Psychiatry 71 Suppl E1 e04.

12. Hawley CJ, Pattinson HA, Quick SJ, Echlin D, Smith V, et al. (1998) A protocol for the pharmacologic treatment of major depression. A field test of a potential prototype. J Affect Disord 47(1-3): 87-96.

13. Ali AM (2018) The relationship between emotional intelligence distress disclosure and psychological distress among Egyptian illicit substance users. J Addict Recovery 1(1002): 1-5.

14. Hritcu L, Ionita R, Postu PA, Gupta GK, Turkez H, et al. (2017) Antidepressant Flavonoids and Their Relationship with Oxidative Stress. Oxid Med Cell Longev.

15. Rink L, Braun C, Bschor T, Henssler J, Franklin J, et al. (2018) Dose Increase Versus Unchanged Continuation of Antidepressants After Initial Antidepressant Treatment Failure in Patients with Major Depressive Disorder: A Systematic Review and Meta-Analysis of Randomized, Double-Blind Trials. J Clin Psychiatry 79(3).

16. Ali AM, Green J (2017) Differential Item Functioning of the Arabic Version of the Depression Anxiety Stress Scale-21 (DASS-21). JOJ Nurse Health Care 4(5).

17. Ali AM, Hendawy AO (2018) Bee Honey as a Potentially Effective Treatment for Depression: A Review of Clinical and Preclinical Findings. JOJ Nurse Health Care 9(2).

18. Turner EH, Matthews AM, Linardatos E, Tell RA, Rosenthal R (2008) Selective publication of antidepressant trials and its influence on apparent efficacy. N Engl J Med 358(3): 252-260.

19. Ebrahim S, Bance S, Athale A, Malachowski C, Ioannidis JPA (2016) Meta-analyses with industry involvement are massively published and report no caveats for antidepressants. Journal of Clinical Epidemiology 70: $155-163$.

20. Hengartner MP, Angst J, Rossler W (2018) Antidepressant Use Prospectively Relates to a Poorer Long-Term Outcome of Depression: Results from a Prospective Community Cohort Study over 30 Years Psychother Psychosom 87(3): 181-183.

21. Deshauer D, Moher D, Fergusson D, Moher E, Sampson M, et al. (2008) Selective serotonin reuptake inhibitors for unipolar depression: a systematic review of classic long-term randomized controlled trials. Cmaj 178(10): 1293-1301.
22. Cappell MS (2004) Colonic Toxicity of Administered Drugs and Chemicals. American Am J Gastroenterol 99(6): 1175-1190.

23. Mirza S (2015) Neurotransmitters: the link between depression, chronic fatigue and chronic pain syndromes. BMJ 350.

24. Macedo D, Filho AJMC, Soares de Sousa CN, Quevedo J, Barichello T, et al. (2017) Antidepressants, antimicrobials or both? Gut microbiota dysbiosis in depression and possible implications of the antimicrobial effects of antidepressant drugs for antidepressant effectiveness. J Affect Disord 208: 22-32.

25. Martin Subero M, Anderson G, Kanchanatawan B, Berk M, Maes M (2016) Comorbidity between depression and inflammatory bowel disease explained by immune-inflammatory, oxidative, and nitrosative stress; tryptophan catabolite; and gut-brain pathways. CNS Spectr 21(2): 184-198.

26. Minguez L, Halm Lemeille MP, Costil K, Bureau R, Lebe JM, et al.(2014) Assessment of cytotoxic and immunomodulatory properties of four antidepressants on primary cultures of abalone hemocytes (Haliotis tuberculata). Aquatic Toxicology 153: 3-11.

27. Read J, Gee A, Diggle J, Butler H (2017) The interpersonal adverse effects reported by 1008 users of antidepressants; and the incremental impact of polypharmacy. Psychiatry Res 256: 423-427.

28. Read J, Gee A, Diggle J, Butler H (2019) Staying on, and coming off, antidepressants: The experiences of 752 UK adults. Addictive Behaviors 88: 82-85.

29. Ciprian A, Furukawa TA, Salanti G, Chaimani A, Atkinson LZ, et al (2018) Comparative efficacy and acceptability of 21 antidepressant drugs for the acute treatment of adults with major depressive disorder: a systematic review and network meta-analysis. The Lancet 391(10128): 1357-1366.

30. Blaber MS, Khan JN, Brebner JA, McColm R (2012). "Lipid Rescue" for Tricyclic Antidepressant Cardiotoxicity. The Journal of Emergency Medicine 43(3): 465-467.

31. Douros A, Ades M, Renoux C (2018) Risk of Intracranial Haemorrhage Associated with the Use of Antidepressants Inhibiting Serotonin Reuptake: A Systematic Review. CNS Drugs 32(4): 321-334.

32. Fu Y, Dai Q Zhu L, Wu S (2018) Antidepressants use and risk of cataract development: a systematic review and meta-analysis. BMC Ophthalmol 18(1): 31

33. Abosi O, Lopes S, Schmitz S, Fiedorowicz JG (2018) Cardiometabolic effects of psychotropic medications. Horm Mol Biol Clin Investig.

34. Elmorsy E, Al Ghafari A, Helaly ANM, Hisab AS, Oehrle B, et al. (2017) Antidepressants Inhibit Pancreatic Beta-Cell Function via Mitochondrial Complex Inhibition. Toxicological Sciences 158(2): 286301.

35. Nazimek K, Strobel S, Bryniarskib P, Kozlowskia M, Filipczak-Bryniarska I, Bryniarski K (2017) The role of macrophages in anti-inflammatory activity of antidepressant drugs. Immunobiology 222(6): 823-830.

36. Tran YH, Schuiling Veninga CCM, Bergman JEH, Groen H, Wilffert B (2017) Impact of Muscarinic M3 Receptor Antagonism on the Risk of Type 2 Diabetes in Antidepressant-Treated Patients: A Case-Controlled Study. CNS Drugs 31(6): 483-493.

37. Ostuzzi G, Matcham F, Dauchy S, Barbui C, Hotopf M (2018) Antidepressants for the treatment of depression in people with cancer Cochrane Database of Systematic Reviews (4).

38. Bennett MI (2010) Effectiveness of antiepileptic or antidepressant drugs when added to opioids for cancer pain: systematic review. Palliative Medicine 25(5): 553-559.

39. Engelmann BJ, Ryan JJ, Farrell NP (2014) Antidepressants and platinum drugs. Anticancer Res 34(1): 509-516. 


\section{Novel Approaches in Drug Designing \& Development}

40. Kane CM, Mulvey MR, Wright S, Craigs C, Wright JM, et al. (2018) Opioids combined with antidepressants or antiepileptic drugs for cancer pain: Systematic review and meta-analysis. Palliat Med 32(1): 276-286.

41. Eisen JN, Irwin J, Quay J, Livnat S (1989) The effect of antidepressants on immune function in mice. Biol Psychiatry 26(8): 805-817.

42. Iishi H, Tatsuta M, Baba M, Taniguchi H (1993) Enhancement by the tricyclic antidepressant, desipramine, of experimental carcinogenesis in rat colon induced by azoxymethane. Carcinogenesis 14(9): 18371840 .

43. LaBella FS, Brandes LJ (1996) Enhancement of tumor growth by drugs with some common molecular actions. Mol Carcinog 16(2): 68-76.

44. Brandes LJ, Arron RJ, Bogdanovic RP, Tong J, Zaborniak CL, et al. (1992). Stimulation of malignant growth in rodents by antidepressant drugs at clinically relevant doses. Cancer Res 52(13): 3796-3800.

45. Dalton SO, Johansen C, Mellemkjaer L, Sorensen HT, McLaughlin JK, et al. (2000) Antidepressant medications and risk for cancer. Epidemiology 11(2): 171-176.

46. Harlow BL, Cramer DW (1995) Self-reported use of antidepressants or benzodiazepine tranquilizers and risk of epithelial ovarian cancer: evidence from two combined case-control studies (Massachusetts, United States). Cancer Causes Control 6(2): 130-134

47. Harlow BL, Cramer DW, Baron JA, Titus Ernstoff L, Greenberg ER (1998) Psychotropic medication use and risk of epithelial ovarian cancer. Cancer Epidemiol Biomarkers Prev 7(8): 697-702.

48. Kelly JP, Rosenberg L, Palmer JR, Rao RS, Strom BL, et al. (1999). Risk of Breast Cancer According to Use of Antidepressants, Phenothizaines, and Anthistamines. American Journal of Epidemiology 150(8): 861868

49. Tsai CJ, Chiu WC, Chen CJ, Chen PC, McIntyre RS, et al.(2018) Antidepressant Prescription and Risk of Lung Cancer: A Nationwide Case-Control Study. Pharmacopsychiatry.

50. Andersohn F, Schade R, Suissa S, Garbe E (2009) Long-term use of antidepressants for depressive disorders and the risk of diabetes mellitus. Am J Psychiatry 166(5): 591-598.

51. Serretti A, Mandelli L (2010) Antidepressants and body weight: a comprehensive review and meta-analysis. J Clin Psychiatry 71(10): 1259-1272.

52. Gadde KM, Allison DB, Ryan DH, Peterson CA, Troupin B, Schwiers ML, et al. (2011) Effects of low-dose, controlled-release, phentermine plus topiramate combination on weight and associated comorbidities in overweight and obese adults (CONQUER): a randomised, placebocontrolled, phase 3 trial. The Lancet 377(9774): 1341-1352.

53. Pij H, Meinders AE (1996) Bodyweight change as an adverse effect of drug treatment. Mechanisms and management. Drug Saf 14(5): 329342

54. Luque CA, Rey JA (2002) The discovery and status of sibutramine as an anti-obesity drug. Eur J Pharmacol 440(2): 119-128.

55. Padwal RS, Majumdar SR (2007) Drug treatments for obesity: orlistat sibutramine, and rimonabant. The Lancet 369(9555): 71-77

56. Hendricks EJ (2017) Off-label drugs for weight management. Diabetes, Metabolic Syndrome and Obesity: Targets and Therapy 10: 223-234.

57. Rubin RR, Ma Y, Marrero DG, Peyrot M, Barrett Connor EL, et al. (2007) Elevated Depression Symptoms, Antidepressant Medicine Use and Risk of Developing Diabetes during the Diabetes Prevention Program. Diabetes Care 31(3): 420-426.

58. Deuschle M (2013) Effects of antidepressants on glucose metabolism and diabetes mellitus type 2 in adults. Curr Opin Psychiatry 26(1): 60 65.
59. Carney RM, Freedland KE, Miller GE, Jaffe AS (2002) Depression as a risk factor for cardiac mortality and morbidity: A review of potential mechanisms. Journal of Psychosomatic Research 53(4): 897-902.

60. Cohen HW, Gibson G, Alderman MH (2000) Excess risk of myocardial infarction in patients treated with antidepressant medications: association with use of tricyclic agents. The American Journal of Medicine 108(1): 2-8.

61. Fisher E, deJarnette J, Shaver J (2018) Are tricyclic antidepressants (TCAs) associated with an increased risk of cardiovascular disease? Evidence-Based Practice 21(3): E21.

62. Robinson DS, Barker, E (1976) Tricyclic antidepressant cardiotoxicity JAMA 236(18): 2089-2090.

63. Niemann JT, Bessen HA, Rothstein RJ, Laks MM (1986) Electrocardiographic criteria for tricyclic antidepressant cardiotoxicity. Am J Cardiol 57(13): 1154-1159.

64. Taylor D (2008) Antidepressant drugs and cardiovascular pathology: a clinical overview of effectiveness and safety. Acta Psychiatr Scand 118(6): 434-442.

65. Mehtonen OP, Aranko K, Malkonen L, Vapaatalo H (1991) A survey of sudden death associated with the use of antipsychotic or antidepressant drugs: 49 cases in Finland. Acta Psychiatr Scand 84(1): 58-64.

66. Andrade C, Sandarsh S, Chethan KB, Nagesh KS (2010) Serotonin reuptake inhibitor antidepressants and abnormal bleeding: a review for clinicians and a reconsideration of mechanisms. J Clin Psychiatry 71(12): 565-1575.

67. Hackam DG, Mrkobrada M (2012) Selective serotonin reuptake inhibitors and brain hemorrhage: a meta-analysis. Neurology 79(18): 1862-1865.

68. Slyepchenko A, Maes M, Jacka FN, Kohler CA, Barichello T, (2017) Gut Microbiota, Bacterial Translocation, and Interactions with Diet: Pathophysiological Links between Major Depressive Disorder and Non-Communicable Medical Comorbidities. Psychother Psychosom 86(1): 31-46.

69. LaChance LR, Ramsey D (2018) Antidepressant foods: An evidencebased nutrient profiling system for depression. World Journal of Psychiatry 8(3): 97-104.

70. Fontani G, Corradeschi F, Felici A, Alfatti F, Migliorini S, et al. (2005) Cognitive and physiological effects of Omega-3 polyunsaturated fatty acid supplementation in healthy subjects. Eur J Clin Invest 35(11): 691-699.

71. Freeman MP, Hibbeln JR, Wisner KL, Davis JM, Mischoulon D, Peet, et al. (2006) Omega-3 fatty acids: evidence basis for treatment and future research in psychiatry. J Clin Psychiatry 67(12): 1954-1967.

72. Lin PY, Su KP (2007) A Meta-Analytic Review of Double-Blind, PlaceboControlled Trials of Antidepressant Efficacy of Omega-3 Fatty Acids. J Clin Psychiatry 68(7): 1057-1062.

73. Appleton KM, Rogers PJ, Ness AR (2010) Updated systematic review and meta-analysis of the effects of $n-3$ long-chain polyunsaturated fatty acids on depressed mood. Am J Clin Nutr 91(3): 757-770.

74. Lin PY, Mischoulon D, Freeman MP, Matsuoka Y, et al. (2012) Are omega-3 fatty acids antidepressants or just mood-improving agents? The effect depends upon diagnosis, supplement preparation, and severity of depression. Mol Psychiatry 17(12): 1161-1163.

75. Laino CH, Fonseca C, Sterin Speziale N, Slobodianik N, Reines A (2010) Potentiation of omega-3 fatty acid antidepressant-like effects with low non-antidepressant doses of fluoxetine and mirtazapine. Eur J Pharmacol 648(1-3): 117-126.

76. Smith DJ, Sarris J, Dowling N, O’Connor M, Ng CH (2018). Adjunctive low-dose docosahexaenoic acid (DHA) for major depression: An openlabel pilot trial. Nutr Neurosci 21(3): 224-228. 
77. Kanchanatawan B, Tangwongchai S, Sughondhabhirom A Suppapitiporn S, Hemrunrojn S, et al. (2018) Add-on Treatment with Curcumin Has Antidepressive Effects in Thai Patients with Major Depression: Results of a Randomized Double-Blind Placebo-Controlled Study. Neurotox Res 33(3): 621-633.

78. He X, Yang L, Wang M, Zhuang X, Huang R, et al. (2017). Targeting the Endocannabinoid/CB1 Receptor System For Treating Major Depression Through Antidepressant Activities of Curcumin and Dexanabinol-Loaded Solid Lipid Nanoparticles. Cell Physiol Biochem 42(6): 2281-2294.

79. Lian L, Xu Y, Zhang J, Yu Y, Zhu N, et al. (2018) Antidepressant-like effects of a novel curcumin derivative J147: Involvement of 5-HT1A receptor. Neuropharmacology 135: 506-513.

80. Sanmukhani J, Satodia V, Trivedi J, Patel T, Tiwari D, et al. (2014) Efficacy and safety of curcumin in major depressive disorder: a randomized controlled trial. Phytother Res 28(4): 579-585.

81. Hosseini M, Zakeri S, Khoshdast S, Yousefian FT, Rastegar M (2012) The effects of Nigella sativa hydro-alcoholic extract and thymoquinone on lipopolysaccharide - induced depression like behavior in rats. Journal of Pharmacy and Bioallied Sciences 4: 219-225.

82. Tuorkey MJ (2017) Therapeutic Potential of Nigella sativa Oil Against Cyclophosphamide-Induced DNA Damage and Hepatotoxicity. Nutrition and Cancer 69(3): 498-504.

83. Kukula Koch W, Koch W, Czernick L, Glowniak K, Asakawa Y (2018) MAO-A Inhibitory Potential of Terpene Constituents from Ginger Rhizomes-A Bioactivity Guided Fractionation. Molecules 23(6).

84. Kwon H, Lee JJ, Lee JH, Cho WK, Gu MJ (2015) Cinnamon and its Components Suppress Vascular Smooth Muscle Cell Proliferation by Up-Regulating Cyclin-Dependent Kinase Inhibitors. The American Journal of Chinese Medicine 43(4): 621-636.

85. Habs M, Binder K, Krauss S, Müller K, Ernst B, et al. (2018) A Balanced Risk-Benefit Analysis to Determine Human Risks Associated with Pyrrolizidine Alkaloids (PA)-The Case of Herbal Medicinal Products Containing St. John's Wort Extracts (SJW). Nutrients 10(7).

86. Khan H, Amin S, Patel S (2018) Targeting BDNF modulation by plant glycosides as a novel therapeutic strategy in the treatment of depression. Life Sci 196: 18-27.

87. Chang SC, Cassidy A, Willett WC, Rimm EB, O’Reilly EJ, et al. (2016) Dietary flavonoid intake and risk of incident depression in midlife and older women. Am J Clin Nutr, 104(3): 704-714.

88. Karim N, Khan I, Abdelhalim A, Khan A, Halim SA (2018). Antidepressant potential of novel flavonoids derivatives from sweet violet (Viola odorata L): Pharmacological, biochemical and computational evidences for possible involvement of serotonergic mechanism. Fitoterapia 128: 148-161.

89. Wang X, Zhang L, Hua L, Xing D, Du L (2010) Effect of Flavonoids in Scutellariae Radix on Depression-Like Behavior and Brain Rewards: Possible in Dopamine System. Tsinghua Science \& Technology 15(4): 460-466.

90. Abu Tarboush HM, Al Kahtani HA, El Sarrage MS (1993) Floral-type identification and quality evaluation of some honey types. Food Chemistry 46(1): 13-17.

91. Alzubier AA, Okechukwu PN (2011) Investigation of Anti-Inflammatory, Antipyretic and Analgesic Effect of Yemeni Sidr Honey. World Academy of Science, Engineering and Technology, 56: 56-52.

92. Alqurashi AM, Masoud EA, Alamin MA (2013) Antibacterial activity of Saudi honey against Gram negative bacteria. Journal of Microbiology and Antimicrobials 5(1): 1-5.
93. Molan PC (1992) The Antibacterial Activity of Honey. Bee World 73(1): 5-28.

94. Nasuti C, Gabbianelli R, Falcioni G, Cantalamessa F (2006) Antioxidative and gastroprotective activities of anti-inflammatory formulations derived from chestnut honey in rats. Nutrition Research 26(3): 130137.

95. Taha MME, Abdelwahab SI, Elsanousi R, Sheikh BY, Abdulla MA, et al. (2015) Effectiveness of Sidr Honey on the prevention of ethanolinduced gatroulcerogenesis: role of antioxidant and antiapoptotic mechanism. Phcog J 7(3): 157-164.

96. Ezz ElArab AM, Girgis SM, Hegazy EM, Abd El Khalek AB (2006) Effect of dietary honey on intestinal microflora and toxicity of mycotoxins in mice. BMC Complement Altern Med 6(1): 6.

97. Al Rahbi B, Zakaria R, Othman Z, Hassan A, Ahmad AH (2014) Protective Effects of Tualang Honey against Oxidative Stress and Anxiety-Like Behaviour in Stressed Ovariectomized Rats. International Scholarly Research Notices p.10.

98. Yildiz O, Karahalil F, Can Z, Sahin H, Kolayli S (2014) Total monoamine oxidase (MAO) inhibition by chestnut honey, pollen and propolis. Enzyme Inhib Med Chem 29(5): 690-694.

99. Fond G, Boukouaci W, Chevalier G, Regnault A, Eberl G, et al. (2015). The "psychomicrobiotic": Targeting microbiota in major psychiatric disorders: A systematic review. Pathologie Biologie 63(1): 35-42.

100. Ng QX, Peters C, Ho CYX, Lim DY, Yeo WS (2018) A meta-analysis of the use of probiotics to alleviate depressive symptoms. J Affect Disord 228: 13-19.

101. Burokas A, Arboleya S, Moloney RD, Peterson VL, Murphy K, et al. (2017) Targeting the Microbiota-Gut-Brain Axis: Prebiotics Have Anxiolytic and Antidepressant-like Effects and Reverse the Impact of Chronic Stress in Mice. Biological Psychiatry 82(7): 472-487.

102. Akkasheh G, Kashani Poor Z, Tajabadi Ebrahimi M, Jafari P, Akbari $\mathrm{H}$, et al. (2016) Clinical and metabolic response to probiotic administration in patients with major depressive disorder: A randomized, double-blind, placebo-controlled trial. Nutrition 32(3): 315-320.

103. Dash S, Clarke G, Berk M, Jacka FN (2015) The gut microbiome and diet in psychiatry: focus on depression. Curr Opin Psychiatry 28(1): $1-6$.

104. Luna RA, Foster JA (2015) Gut brain axis: diet microbiota interactions and implications for modulation of anxiety and depression. Current Opinion in Biotechnology 32: 35-41.

105. Claesson MJ, Jeffery IB, Conde S, Power SE, O'Connor EM, et al. (2012) Gut microbiota composition correlates with diet and health in the elderly. Nature 488: 178-184.

106. De Filippis F, Pellegrini N, Vannini L, Jeffery IB, La Storia A, et al. (2016) High-level adherence to a Mediterranean diet beneficially impacts the gut microbiota and associated metabolome. Gut 65(11): 1812-1821.

107. Ashton AK, Jamerson BD, Weinstein LW, Wagoner C (2005) Antidepressant-related adverse effects impacting treatment compliance: Results of a patient survey. Curr Ther Res Clin Exp 66(2): 96-106.

108. Ho SC, Jacob SA, Tangiisuran B (2017) Barriers and facilitators of adherence to antidepressants among outpatients with major depressive disorder: A qualitative study. PLoS One 12(6): e0179290 


\section{Your next submission with Juniper Publishers} will reach you the below assets

- Quality Editorial service

- Swift Peer Review

- Reprints availability

- E-prints Service

- Manuscript Podcast for convenient understanding

- Global attainment for your research

- Manuscript accessibility in different formats ( Pdf, E-pub, Full Text, Audio)

- Unceasing customer service

Track the below URL for one-step submission https://juniperpublishers.com/online-submission.php 